\title{
Glaucoma Education Intervention: Using a Trained Volunteer to Improve Patient Understanding and Compliance
}

\author{
Mital Shah, Asifa Shaikh* \\ Department of Ophthalmology, Stoke Mandeville Hospital, Aylesbury, UK \\ Email address: \\ Asifa.Shaikh@buckshealthcare.nhs.uk (A. Shaikh) \\ ${ }^{*}$ Corresponding author
}

To cite this article:

Mital Shah, Asifa Shaikh. Glaucoma Education Intervention: Using a Trained Volunteer to Improve Patient Understanding and Compliance. International Journal of Ophthalmology \& Visual Science. Vol. 3, No. 2, 2018, pp. 27-31. doi: 10.11648/j.ijovs.20180302.13

Received: June 28, 2018; Accepted: July 23, 2018; Published: August 17, 2018

\begin{abstract}
The aim of this study is to assess patients' experience and acceptability of an educational intervention run by volunteers to provide information about glaucoma, with the goal of improving patients' understanding about glaucoma. Clinicians referred patients attending glaucoma clinics at one trust to volunteers for education about glaucoma, surgical or laser interventions and eye drop technique. Patients were subsequently contacted by telephone to complete a satisfaction survey with five responses ranging from strongly disagree to strongly agree. 68 patients were referred to the volunteers over a 7 month period. $27(40 \%)$ were interviewed by telephone. All were happy with their experience of the volunteer service with 23 patients $(57.5 \%)$ strongly agreeing that they were comfortable speaking to a volunteer, $18(45 \%)$ strongly agreeing that the volunteer was able to answer their questions and explain the condition properly and $22(55 \%)$ strongly agreeing that speaking with the volunteer was a positive experience. 17 (42.5\%) strongly agreed that they had better knowledge and understanding about glaucoma and $22(55 \%)$ strongly agreed that they understood the importance regular therapy. Of the 17 patients surveyed about eye drop technique, $14(82.4 \%)$ strongly agreed they were more confident with their technique. Patients were comfortable and accepting of the volunteer scheme and reported better understanding of glaucoma. Using volunteers to educate patients helps clinicians and improves compliance.
\end{abstract}

Keywords: Glaucoma, Patient Education, Compliance, Adherence, Volunteers, Non-Medical Assistants

\section{Introduction}

Glaucoma is one of the leading causes of blindness globally [1]. In 2010, The World Health Organisation (WHO) estimated that 39 million people in the world were blind, and $8 \%$ of these (over 3 million people) were due to glaucoma [1]. In the United Kingdom approximately $10 \%$ of blindness registrations are as a result of glaucoma, and it accounts for over 1 million hospital eye service visits annually [2].

Treatment of glaucoma prevents visual impairment and involves the use of medical and surgical approaches. Current treatment of glaucoma is centred on a reduction of intraocular pressure. Medical treatment of glaucoma using eye drops is the most common form of initial treatment and requires good compliance. As glaucoma is initially asymptomatic, noncompliance with glaucoma medications is common. Persistence with taking medications has been reported to vary from $20-64 \%[3,4]$.

There are many different reasons for reduced compliance and lack of information about glaucoma has shown to be a causative factor [5-7], specifically if patients do not understand that risk of visual loss is increased if they do not take their medication as prescribed by their specialist [8]. Studies have shown that a tailored approach centred around the patient with initial education about glaucoma and the importance of taking the eye drops could help improve motivation and hence compliance [8].

The National Institute of Clinical Excellence (NICE) guideline for glaucoma describes a typical patient care pathway and states that healthcare professionals should provide information for patients and family members [2]. Educating patients about their diagnosis and plan of care is 
important, especially as a number of studies have suggested that educational interventions improve compliance [9-12]. Patient information leaflets on glaucoma and its treatment may be sufficient in a lot of cases. However, this information often also needs to be relayed verbally and a large number of patients benefit from having this message reinforced from time to time. Younger patients may have time constrains with family and work commitments and older patients may have physical barriers to good compliance with drop wastage leading to variable or poor compliance. Moreover with time, compliance with medications may falter due to side effects of medications and lack of symptoms secondary to glaucoma itself. Experience from working in glaucoma clinics shows that patients respond favourably to a more discussion-based conversation and prefer a personalised one-on-one consultation with their specialist or health educator [13]. Clinicians have time constraints to deliver effective educational interventions and trained volunteers can offer an alternative opportunity to educate patients, which may help improve compliance and also be cost effective.

This study was set up to assess patients' experience and acceptability of an educational intervention from a group of trained volunteers to provide information about glaucoma and its treatment.

\section{Material and Methods}

Over a 7 month period at one NHS trust, clinicians referred patients attending glaucoma clinics to one of the trained volunteers for education about glaucoma, its treatment including surgical or laser interventions and eye drop technique. The clinician indicated the type of advice the volunteer was to give the patient via a request form.

Nine glaucoma volunteers had training sessions provided by a consultant ophthalmologist with a special interest in glaucoma and a representative of the International Glaucoma Association (IGA) about glaucoma, its various treatment options and the importance of adherence to medications. All volunteers were taught appropriate techniques for administering eye drops correctly including demonstration and practice of the IGA compliance briefcase. The volunteers were also provided with information about the different laser and surgical treatments that glaucoma patients could have and were also provided with information about the pathway to these treatment options and the pre and post-operative care required. They were also provided with departmental leaflets relating to these treatments to hand over to patients. In addition, they also had leaflets provided by the IGA with information about the different types of glaucoma. The Royal National Institute of Blind People (RNIB) also presented a training session for the volunteers on how to support patients in the eye clinic.

The volunteer had a discussion with the patients after their consultation with an ophthalmologist, with the aim of stimulating interaction about glaucoma and discussing any concerns they may have about its treatment and management. Over the 7 month period, 68 patients were referred by clinical staff to the clinic volunteers. 49 patients were subsequently contacted by telephone to complete a satisfaction survey, which included five responses ranging from strongly agree to strongly disagree.

\section{Results}

Of the 49 patients contacted by telephone, 27 (55\%) were available to answer the call and take part in the survey.

All were happy with their experience of the volunteer service with 23 patients $(57.5 \%)$ strongly agreeing that they were comfortable talking to a volunteer, 18 (45\%) strongly agreeing that the volunteer was able to answer their questions and explain the condition properly and 22 (55\%) strongly agreeing that speaking with the volunteer was a positive experience (Figure 1).

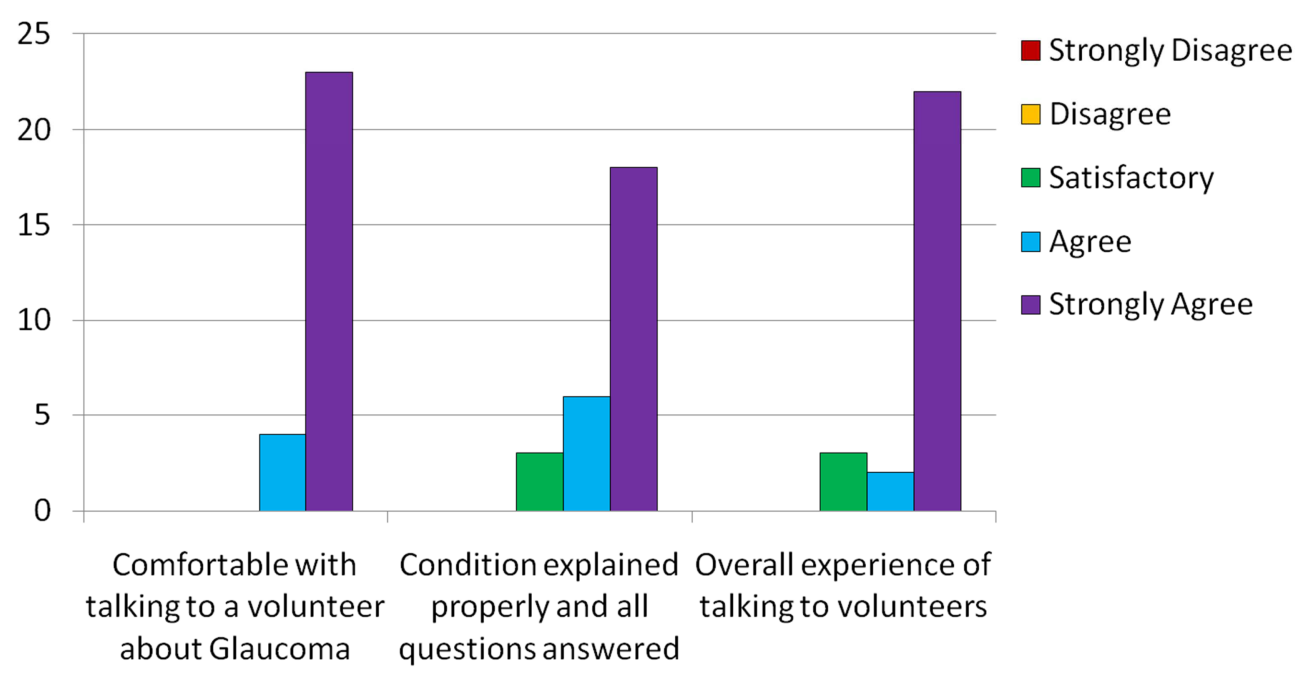

Figure 1. Patient experience of the volunteer service.

17 (42.5\%) strongly agreed that they had better knowledge and understanding about glaucoma and 22 (55\%) strongly agreed that they understood the importance regular therapy (Figure 2). 


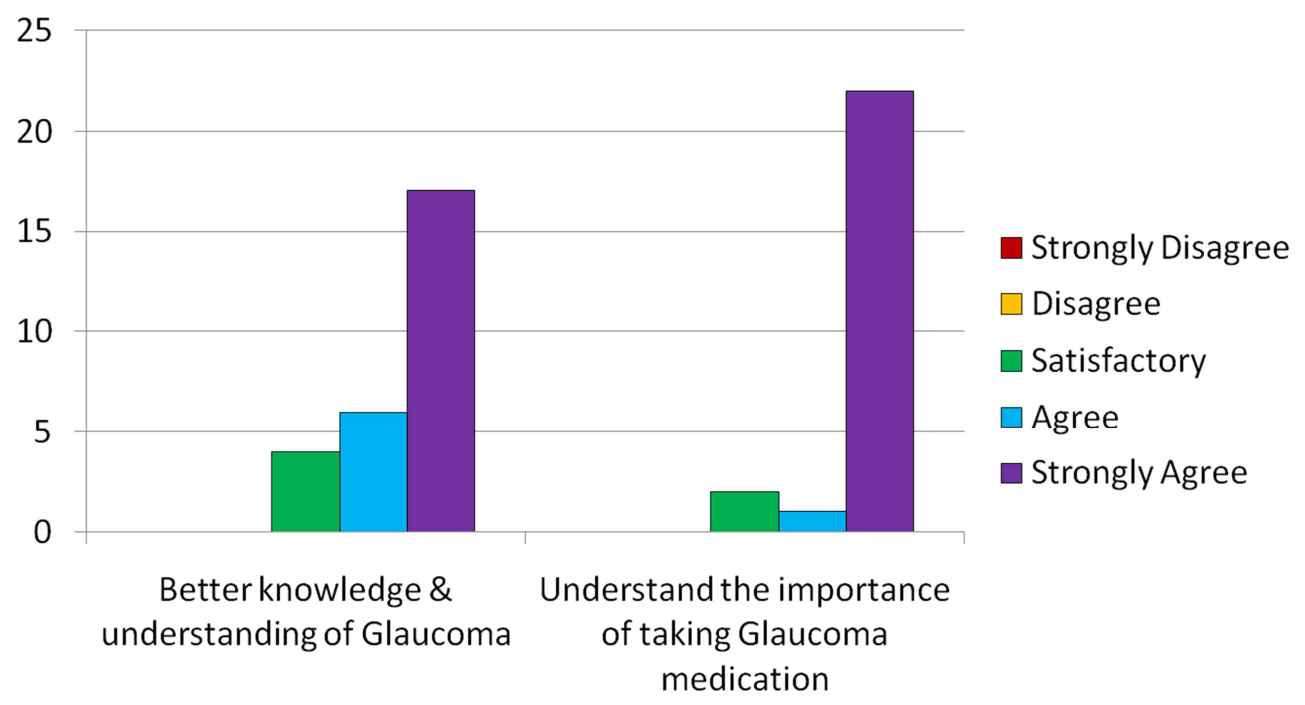

Figure 2. Patient experience of the volunteer advice.

Of the 17 patients surveyed about eye drop technique, $14(82.4 \%)$ strongly agreed they were more confident with their technique (Figure 3).

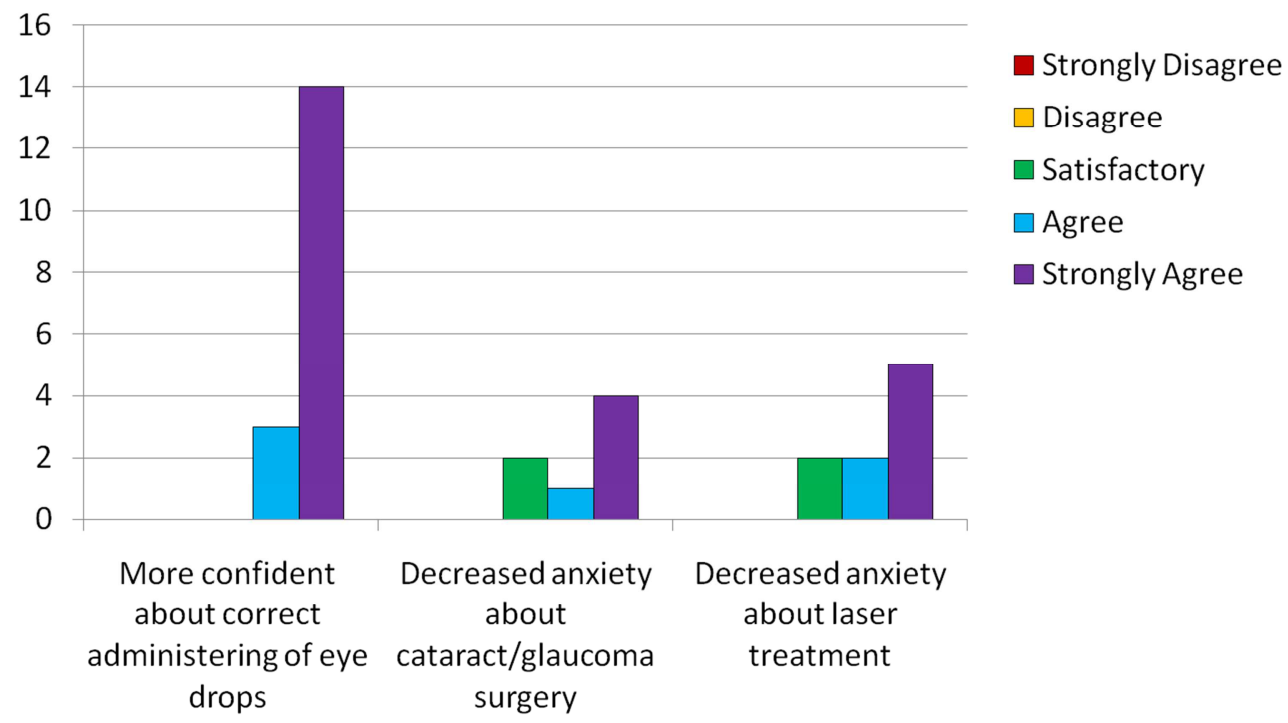

Figure 3. Patient experience of the volunteer advice regarding specific treatements.

\section{Discussion}

This study assessed the acceptability of trained volunteers to provide information to patients on glaucoma and its treatment using patient reported outcomes. Our results show that such trained volunteers working within glaucoma clinics are not only acceptable to patients, but help to provide patients with better understanding of glaucoma and its treatment including the ability to instil eye drops correctly.

Various studies have demonstrated that patient education interventions help to improve compliance. Muir et al. showed patients with lower literacy levels were less compliant with taking their medications. They concluded interventions targeted towards improving health literacy in glaucoma patients might increase subjects' compliance to a therapeutic regime [14]. Juzych et al. assessed the relationship between health literacy and compliance in patients with glaucoma. Their study showed patients with poor health literacy had poorer compliance [15]. Other studies have also used interventions in the form of educational videos and telephone reminders to improve adherence with glaucoma medications [12]. Glanz et al. showed improvement in compliance with glaucoma treatment using a tailored automated telephone intervention and printed materials [16]. In 2012, Muir et al. conducted a study using educational videos and printed materials on glaucoma tailored to the participants' literacy level and showed that patients in the intervention group missed less days of medication compared to the control group [17].

The Glaucoma Adherence and Persistency Study is a series of publications evaluating pharmacy claims, patient and physician interviews and physician charts to determine 
medication compliance $[8,18,19]$. Lower rates of compliance were found in patients whose only understanding of glaucoma was from their doctor, those who did not believe that not taking medication would lead to reduced vision and those often travelling away from home. Patients given follow up telephone calls had higher rates of compliance.

A Cochrane review on the Interventions for improving adherence to ocular hypotensive therapy concluded that 'educating patients about their condition, teaching them how to instil eye drops, providing reminders and working individually with patients to help them manage their eye drop routines have provided positive findings for improving adherence levels' [20]. For many patients the main motivation for adherence is the fear of going blind along with a belief in eye drop efficacy $[5,7]$.

Busy glaucoma clinics are already struggling with patient numbers and with an increasing aging population their workload is only set to increase. Clinicians working in these busy clinics do not always have the time to provide adequate patient education. Moreover it has been shown that compliance with therapy is often poor when the information has been provided by the doctor alone and that compliance improves when the information is repeated and reinforced by other means and methods [8, 18, 19]. Adequate and timely patient education in glaucoma and its treatment is an important factor in improving glaucoma medication compliance. Patients require individualised information. Therefore, it is important to look at and implement alternative, effective, but also an acceptable method of educating patients about glaucoma, its implications, the importance of regular therapy, good compliance, regular follow up and the possibility of permanent visual loss in cases of poor compliance.

To our knowledge, this is the first study of its kind using non-medical volunteers to provide patient information on glaucoma, its treatment and demonstration of eye drop technique. Our study shows that not only were patients very happy receiving information from volunteers working as a part of the glaucoma clinic team, but that they felt they had a better understanding about glaucoma and understood the importance of regular use of glaucoma medications. Furthermore, patients reported being much more confident with the use of their eye drops following this consultation. The use of non-medical personnel for the provision of this information may also offer economic benefits compared to a model using other healthcare professionals like nurses. The emphasis of the volunteer should be at the point of diagnosis and in cases where poor compliance is suspected. Moreover, a study by our group showed that compliance tends to be poor in younger age groups and in those over the age of 90 [21]. Educational interventions specifically targeted to these groups of patients can be provided by the clinic volunteers; emphasising the risk of visual loss in the patient's expected lifespan, possibly resulting in loss of earning and driving licence in the younger patients and helping with the use of compliance aids in the older age groups. We also found that educating and enlisting the help of family members helped to improve compliance with medications in the elderly population; family members could be enlisted to help with instilling or observing the use of eye drops. Sometimes a regular phone call to remind a relative with failing memory to use their drops was found to be useful. Additionally, we found that patients or their relatives tend to have a lot of questions they want to ask about laser treatment or surgery after the consultation with the doctor is completed. Most of these questions related to patient concerns and anxiety about the treatment being advised. We extended the service provided by our clinic volunteers by referring these patients and found that the volunteers were able to address most of these concerns. The patients concerned found that they were able to have a more relaxed and informal discussion with the volunteer outside of the consultation with the doctor and felt they were able to discuss their concerns more freely. This consultation was not designed to provide medical information like indications, risks and benefits of treatment but to allay patient fears and concerns about the pathway to treatment and postoperative care.

Patient education should centre on prognosis of glaucoma, eye drop application techniques, scheduling issues and consequences of poor compliance, all of which can improve glaucoma medication adherence [22]. It is likely that educational consultations at the point of diagnosis are invaluable in helping patients learn, understand and take ownership about their condition. Individualising educational material and providing information tailored to the needs of each patient according to their lifestyle and age and focusing on specific adherence concerns can lead to improved confidence with eye drop technique. Studies have shown that this technique can be more effective than the use of standard materials in other aspects of health prevention, for example, smoking cessation [23].

\section{Conclusion}

Our study has shown that trained, non-medical assistants can be an invaluable asset in the provision of patient educational intervention and may improve patient compliance with glaucoma medications. Furthermore, with appropriate training, the role of these assistants can be extended to provide information, answer questions and to allay patient concerns and fears about surgical and laser treatments.

\section{Conflict of Interest}

The authors declare no conflict of interest.

\section{Acknowledgements}

The authors would like to thank Antonia Tse, Deborah Dow, Jane Diggory and BucksVision, Visual Impairment Charity for their contributions towards this manuscript. 


\section{References}

[1] Pascolini D, Mariotti SP. Global estimates of visual impairment: 2010. Br J Ophthalmol. 2012; 96 (5): 614-618.

[2] National Collaborating Centre for Acute Care (UK). Glaucoma: Diagnosis and Management of Chronic Open Angle Glaucoma and Ocular Hypertension. London: National Collaborating Centre for Acute Care (UK); 2009.

[3] Spooner JJ, Bullano MF, Ikeda LI, Cockerham TR, Waugh WJ, Johnson T, et al. Rates of discontinuation and change of glaucoma therapy in a managed care setting. Am J Manag Care. 2002; 8 (10 Suppl): S262-70.

[4] Dasgupta S, Oates V, Bookhart BK, Vaziri B, Schwartz GF, Mozaffari E. Population-based persistency rates for topical glaucoma medications measured with pharmacy claims data. Am J Manag Care. 2002; 8 (10 Suppl): S255-61.

[5] Lacey J, Cate H, Broadway DC. Barriers to adherence with glaucoma medications: a qualitative research study. Eye (Lond). 2009; 23 (4): 924-932.

[6] Stryker JE, Beck AD, Primo SA, Echt KV, Bundy L, Pretorius $\mathrm{GC}$, et al. An exploratory study of factors influencing glaucoma treatment adherence. Journal of Glaucoma. 2010; 19 (1): 66-72.

[7] Tsai JC, McClure CA, Ramos SE, Schlundt DG, Pichert JW. Compliance Barriers in Glaucoma: A Systematic Classification. Journal of Glaucoma. 2003; 12 (5): 393.

[8] Friedman DS, Hahn SR, Gelb L, Tan J, Shah SN, Kim EE, et al. Doctor-patient communication, health-related beliefs, and adherence in glaucoma results from the Glaucoma Adherence and Persistency Study. Ophthalmology. 2008; 115 (8): 1320 7-1327. e1-3.

[9] Rendell J. Effect of health education on patients' beliefs about glaucoma and compliance. Insight. 2000; 25 (4): 112-118.

[10] Cook PF, Bremer RW, Ayala AJ, Kahook MY. Feasibility of motivational interviewing delivered by a glaucoma educator to improve medication adherence. Clin Ophthalmol. 2010; 4: $1091-1101$.

[11] Chen X, Chen Y, Sun X. Notable role of glaucoma club on patients' knowledge of glaucoma. Clin. Experiment. Ophthalmol. 2009; 37 (6): 590-594.

[12] Okeke CO, Quigley HA, Jampel HD, Ying G-S, Plyler RJ, Jiang $\mathrm{Y}$, et al. Interventions improve poor adherence with once daily glaucoma medications in electronically monitored patients. Ophthalmology. 2009; 116 (12): 2286-2293.

[13] Rosdahl JA, Swamy L, Stinnett S, Muir KW. Patient education preferences in ophthalmic care. Patient preference and adherence. 2014; 8: 565-574.

[14] Muir KW, Santiago-Turla C, Stinnett SS, Herndon LW, Allingham RR, Challa P, et al. Health literacy and adherence to glaucoma therapy. Am. J. Ophthalmol. 2006; 142 (2): 223 226.

[15] Juzych MS, Randhawa S, Shukairy A, Kaushal P, Gupta A, Shalauta N. Functional health literacy in patients with glaucoma in urban settings. Arch Ophthalmol. 2008; 126 (5): 718-724.

[16] Glanz K, Beck AD, Bundy L, Primo S, Lynn MJ, Cleveland J, et al. Impact of a health communication intervention to improve glaucoma treatment adherence. Results of the interactive study to increase glaucoma adherence to treatment trial. Arch Ophthalmol. 2012; 130 (10): 1252-1258.

[17] Muir KW, Ventura A, Stinnett SS, Enfiedjian A, Allingham RR, Lee PP. The influence of health literacy level on an educational intervention to improve glaucoma medication adherence. Patient Educ Couns. 2012; 87 (2): 160-164.

[18] Friedman DS, Quigley HA, Gelb L, Tan J, Margolis J, Shah $\mathrm{SN}$, et al. Using pharmacy claims data to study adherence to glaucoma medications: methodology and findings of the Glaucoma Adherence and Persistency Study (GAPS). Invest. Ophthalmol. Vis. Sci. 2007; 48 (11): 5052-5057.

[19] Quigley HA, Friedman DS, Hahn SR. Evaluation of practice patterns for the care of open-angle glaucoma compared with claims data: the Glaucoma Adherence and Persistency Study. Ophthalmology. 2007; 114 (9): 1599-1606.

[20] Waterman H, Evans JR, Gray TA, Henson D, Harper R. Interventions for improving adherence to ocular hypotensive therapy. Waterman H (ed). Cochrane Database Syst Rev. 2013; 4: CD006132.

[21] Tse AP, Shah M, Jamal N and Shaikh A. Glaucoma treatment adherence at a United Kingdom general practice. Eye. 2016; 30: $1118-1122$.

[22] Newman-Casey PA, Weizer JS, Heisler M, Lee PP, Stein JD. Systematic review of educational interventions to improve glaucoma medication adherence. Semin Ophthalmol. 2013; 28 (3): 191-201.

[23] Strecher VJ. Computer-tailored smoking cessation materials: a review and discussion. Patient Educ Couns. 1999; 36 (2): 107-117. 